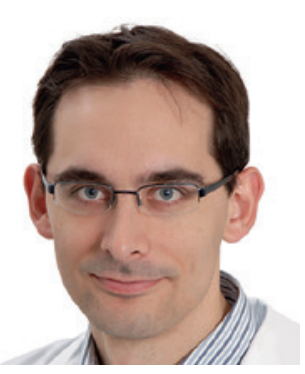

\title{
Gewebsresidente CD8+ T-Zellen als therapeutisches Ziel bei der Checkpunkttherapie-assoziierten Immunenterokolitis
}

\section{Bertram Bengsch}

Universitätsklinikum Freiburg, Innere Medizin II, Gastroenterologie, Hepatologie, Endokrinologie und Infektiologie, Freiburg im Breisgau, Deutschland

Abstract aus Sasson SC, Slevin SM, Cheung VTF, et al.: Interferon-gamma-producing CD8 ${ }^{+}$tissue resident memory T cells are a targetable hallmark of immune checkpoint inhibitor-colitis. Gastroenterology 2021;161:1229-1244.e9.

\section{Keywords}

Checkpoint Colitis · Immunotherapy Colitis · Tofacitinib

Ulcerative Colitis

\begin{abstract}
Background \& Aims: The pathogenesis of immune checkpoint inhibitor (ICI)-colitis remains incompletely understood. We sought to identify key cellular drivers of $\mathrm{ICl}$-colitis and their similarities to idiopathic ulcerative colitis, and to determine potential novel therapeutic targets.
\end{abstract}

Methods: We used a cross-sectional approach to study patients with $\mathrm{ICl}$-colitis, those receiving $\mathrm{ICl}$ without the development of colitis, idiopathic ulcerative colitis, and healthy controls. A subset of patients with $\mathrm{ICl}$-colitis were studied longitudinally. We applied a range of methods, including multiparameter and spectral flow cytometry, spectral immunofluorescence microscopy, targeted gene panels, and bulk and single-cell RNA sequencing.
Results: We demonstrate $C D 8^{+}$tissue resident memory $T\left(T_{R M}\right)$ cells are the dominant activated $\mathrm{T}$ cell subset in $\mathrm{ICl}$-colitis. The pattern of gastrointestinal immunopathology is distinct from ulcerative colitis at both the immune and epithelial-signaling levels. $C D 8^{+} T_{\text {RM }}$ cell activation correlates with clinical and endoscopic $\mathrm{ICl}$-colitis severity. Single-cell RNA sequencing analysis confirms activated $\mathrm{CD}^{+} \mathrm{T}_{\mathrm{RM}}$ cells express high levels of transcripts for checkpoint inhibitors and interferon-gamma in $\mathrm{ICl}$ colitis. We demonstrate similar findings in both anti-CTLA-4/ PD-1 combination therapy and in anti-PD-1 inhibitor-associated colitis. On the basis of our data, we successfully targeted this pathway in a patient with refractory $\mathrm{ICl}$-colitis, using the JAK inhibitor tofacitinib.

Conclusions: Interferon gamma-producing $\mathrm{CD}^{+} \mathrm{T}_{\mathrm{RM}}$ cells are a pathological hallmark of $\mathrm{ICl}$-colitis and a novel target for therapy. (c) 2021 The Authors 


\section{Transfer in die Praxis}

\section{Hintergrund}

Eine Enterokolitis ist die häufigste gastrointestinale Nebenwirkung der Immuncheckpunkttherapien. Je nach Therapieschema kann sie bei bis zu 40\% der behandelten Patient*innen auftreten (Doppelimmuntherapie mit PD-1- und CTLA-4-Inhibitoren), seltener bei der Monotherapie mit Anti-PD-1- (etwa 2-5\%) oder AntiCTLA-4-Antikörpern (etwa 10\%). Sie manifestiert sich meist durch Diarrhöen, kann jedoch schwerwiegender mit abdominellen Schmerzen, Blutungen, Ulzerationen, Fieber, Fisteln, Perforationen, Peritonitis und septischen Krankheitsbildern einhergehen. Niedriggradige Nebenwirkungen können meist ausreichend symptomorientiert, z.B. mit Loperamid, behandelt werden, bei stärkeren Nebenwirkungen ist eine Behandlung mit Kortikosteroiden indiziert. In der Regel kann eine frühzeitige systemische immunsuppressive Behandlung mit Kortikosteroiden lebensbedrohliche Verläufe mit intestinaler Perforation und Peritonitis verhindern. Im Fall eines steroidrefraktären Verlaufes werden als zusätzliche Immunsuppressiva in Analogie zur Behandlung chronisch-entzündlicher Darmerkrankungen meist Biologika wie AntiTNF-Antikörper oder Anti-a4ß7-Antikörper eingesetzt. Bisherige Studien haben auf intestinale Immunzellen, vor allem CD4- und CD8-positive T-Zellen, als wesentliche Vermittler der Kolitis hingewiesen. Das Verständnis der Immunpathogenese dieser autoimmunvermittelten Erkrankung und die Evidenz für die immunsuppressiven Therapiestrategien sind jedoch noch sehr eingeschränkt.

\section{Studienzusammenfassung und Kommentar}

In der aktuellen Arbeit von Sasson et al. haben die Kolleg*innen der Universität Oxford eine umfangreiche Immunanalyse bei Patient*innen mit Immunenterokolitis (IEK) durchgeführt. Es wurden 15 Patient*innen mit Doppelimmuntherapie- und 6 Patient*innen mit Anti-PD-1-Monotherapie-assoziierter Enterokolitis eingeschlossen, als Kontrollgruppen dienten Patient*innen mit gematchter Immuntherapie ohne gastrointestinale Nebenwirkungen, Patient*innen mit aktiver Colitis ulcerosa und gesunde Kontrollen. Im Rahmen einer Sigmoidoskopie wurden Biopsien entnommen, die mit Einzelzelltranskriptionsanalysen, Panelex- pressionsanalysen, Durchflusszytometrie und hochauflösenden histologischen Methoden charakterisiert wurden. Es zeigte sich eine deutliche Anreicherung aktivierter gewebsresidenter CD8+ T-Zellen ( $T_{\text {RM }}$-Zellen) bei den Patient*innen mit IEK. Diese $T_{\text {RM }}$-Zellen exprimieren das Integrin aE (CD103), das an Liganden auf Epithelzellen bindet, sie exprimieren aber auch Immuncheckpunkte wie PD-1 und CTLA-4, was ihre aberrante Aktivierung im Kontext der Immuntherapie erklären könnte. Die aktivierten $T_{\text {RM }}$-Zellen fanden sich in den Schleimhautkrypten und der Lamina propria, passend zu ihrem gewebsresidenten Phänotyp. Interessanterweise korrelierte die Aktivierung der $\mathrm{T}_{\mathrm{RM}}$-Zellen mit dem endoskopischen Befund, sie war jedoch deutlich ausgeprägter als bei Patient*innen mit Colitis ulcerosa und ähnlicher Krankheitsaktivität. Die Transkriptomanalysen zeigten ebenfalls distinkte Unterschiede zwischen der Entzündungsaktivität bei der IEK und der Colitis ulcerosa. Insbesondere fiel eine deutliche Aktivierung des Interferonsignalwegs auf, der durch den JAK-STAT-Signalweg induziert wird, bei ebenfalls vorhandener, jedoch weniger stark ausgeprägter Produktion von TNF. Diese Untersuchungen wiesen auf die relevanten Signalwege pathogenetisch relevanter $T_{\mathrm{RM}^{-}}$ Zellen hin. Die Autoren konnten diese Erkenntnisse dann bei einem Patienten translational anwenden, bei dem eine schwere steroidrefraktäre IEK nach 2 Zyklen Chemoimmuntherapie (Pembrolizumab, Carboplatin und Permetrexed) bei einem metastasierten nichtkleinzelligen Lungenkarzinom auftrat. Auch hochdosierte intravenöse Steroidgabe und 2 Gaben von Infliximab zeigten kein Ansprechen. Ebenfalls führte eine probatorische Stuhltransplantation nur zu einer geringen Besserung, und auch nach 3-monatiger Behandlung der IEK bestand eine klinisch und endoskopisch steroidrefraktäre Kolitis mit weiterhin stark aktivierten $\mathrm{T}_{\mathrm{RM}}$-Zellen. Die Kolleg*innen führten daraufhin eine Behandlung mit dem JAK-Inhibitor Tofacitinib durch (2-mal 10 mg/Tag). Hierunter kam es zu einem raschen klinischen und endoskopischen Ansprechen, insbesondere zeigte sich auch eine deutliche Reduktion der Aktivierung der $\mathrm{T}_{\mathrm{RM}}$-Zellen. Nach 6-wöchiger Tofacitinib-Gabe konnte der Patient seine Chemotherapie wiederaufnehmen, es kam zu keinem Wiederauftreten der IEK.

Tab. 1. CTCAE-v5-Graduierung der gastrointestinalen Toxizität

\begin{tabular}{|c|c|c|c|c|c|}
\hline & Grad 1 & Grad 2 & Grad 3 & Grad 4 & Grad 5 \\
\hline \multirow[t]{3}{*}{ Diarrhö } & \multirow{3}{*}{$\begin{array}{l}\text { Zunahme der } \\
\text { Stuhlfrequenz <4/Tag } \\
\text { über Baseline }\end{array}$} & $\begin{array}{l}\text { Stuhlfrequenzzunahme 4-6/Tag } \\
\text { über Baseline }\end{array}$ & $\begin{array}{l}\text { Stuhlfrequenzzunahme }>7 / \text { Tag über } \\
\text { Baseline }\end{array}$ & \multirow[t]{3}{*}{$\begin{array}{l}\text { lebensbedrohliche } \\
\text { Komplikationen }\end{array}$} & \multirow[t]{3}{*}{ Todesfolge } \\
\hline & & $\begin{array}{l}\text { geringe Einschränkung der } \\
\text { Alltagstätigkeit }\end{array}$ & $\begin{array}{l}\text { schwere Einschränkung der } \\
\text { Alltagstätigkeit }\end{array}$ & & \\
\hline & & & stationäre Aufnahme & & \\
\hline Enterokolitis & asymptomatisch & $\begin{array}{l}\text { abdominelle Schmerzen, Blut-/ } \\
\text { Schleimbeimengungen im Stuhl }\end{array}$ & $\begin{array}{l}\text { schwere abdominelle Schmerzen, } \\
\text { Fieber, lleussymptomatik, Peritonismus }\end{array}$ & $\begin{array}{l}\text { lebensbedrohliche } \\
\text { Komplikationen }\end{array}$ & Todesfolge \\
\hline
\end{tabular}




\section{Fazit für die Praxis}

Steroidrefraktäre Verläufe stellen eine Herausforderung für die Behandlung von Patient*innen mit immunvermittelten Nebenwirkungen dar. Häufig führen sie zu hoher Morbidität, kumulativ hohen Steroiddosen mit signifikanten Nebenwirkungen sowie langen Phasen signifikanter Immunsuppression durch die Anwendung ergänzender Immunsuppressiva und gefährden so den onkologischen Therapieerfolg der Immuntherapie. In der vorgestellten Arbeit werden aktivierte $T_{\mathrm{RM}}$-Zellen als pathogenetisch relevante Immunzellpopulation und möglicherweise nutzbare Biomarker identifiziert, deren Aktivierung und Zytokinproduktion an den JAK-STAT-Signalweg gekoppelt ist. JAK-Inhibitoren wie Tofacitinib erscheinen dadurch als eine rationale Therapieoption bei Patient*innen mit steroidrefraktärem Verlauf einer IEK; in der Tat zeigte sich bei dem behandelten Patienten eine rasche klinische Besserung sowie ein Abfall der Biomarker. Auch wenn diese Biomarker und das therapeutische Vorgehen in größeren Studien noch validiert werden müssen, weist die Studie auch auf die grundsätzliche Relevanz der endoskopischen Untersuchung bei Patient*innen mit IEK hin. Patient*innen mit IEK und höhergradi- ger klinischer Symptomatik (CTCAE-Graduierung $\geq 2$; Tab. 1) sollten daher niederschwellig endoskopisch und bioptisch untersucht werden. Bei fehlendem Ansprechen auf Hochdosissteroide und Infliximab erscheint Tofacitinib als eine rationale Therapieoption. Die Therapieentscheidung sollte idealerweise im interdisziplinären Austausch mit den beteiligten Fachgebieten erfolgen. An unserer Institution (Uniklinik Freiburg) hat sich hierfür ein separates Tumorboard für immunvermittelte Nebenwirkungen etabliert. Ferner wird der systematische Einschluss dieser komplizierten Patient*innen in prospektive immunsuppressive Therapiestudien notwendig sein, um eine bessere Datengrundlage und Evidenz für die Auswahl der Zweit- und Drittlinienimmunsuppressiva zu schaffen.

\section{Disclosure Statement}

The author has no conflict of interest.

Korrespondenz an:

Prof. Dr. Dr. Bertram Bengsch, bertram.bengsch@uniklinik-freiburg.de 